\title{
Clinical characteristics and treatment outcomes of severe asthma patients with a history of multiple biologic drugs use
}

\author{
Tomohiro Akaba, Mitsuko Kondo, Soshi Muramatsu, Kazuhiro Abe, Fumi Kobayashi, Azusa Miyoshi, Osamitsu Yagi, \\ Kiyoshi Takeyama, Etsuko Tagaya
}

\begin{abstract}
Background: Asthma control has been shown to improve after clinical use of molecular-targeted biologic drugs. Although most patients have shown favorable responses to biologic drugs, some individuals need to switch to another biologic drug. To date, limited data are available regarding patients who received multiple biologic drugs.
\end{abstract}

Objective: We aimed to evaluate the characteristics and outcomes of patients treated with multiple biologic drugs.

Methods: We reviewed severe asthma patients who received biologic drugs between May 2009 and September 2019. Clinical characteristics of patients and changes in annualized asthma exacerbation rates, asthma control test (ACT), and oral corticosteroid (OCS) dose, before and after the use of the final biologic drug, were evaluated.

Results: Of the 105 patients who received biologic drugs, 20 patients received multiple biologic drugs. Twelve patients received two biologic drugs, six received three, and two received four. Patients who received multiple biologic drugs tended to have a significantly higher number of allergic or eosinophilic airway comorbidities (allergic rhinitis: $p=0.02$, chronic rhinosinusitis with nasal polyps: $p<0.001$ ). Approximately half of the patients changed to different treatments due to uncontrolled comorbidities. Annualized exacerbation rates, ACT, and OCS dose significantly improved after the latest biologic drug use $(p=0.035, p<0.001$, and $p=0.038$, respectively).

Conclusion: The results of this study indicated that allergic and eosinophilic airway comorbidities should be considered during the selection of biologic drugs. Furthermore, most patients who received multiple biologic drugs achieved disease control after switching to the optimal biologic drug.

Key words: severe asthma, biologic drug, allergic comorbidity, allergic rhinitis, chronic rhinosinusitis with nasal polyps

\section{From:}

Department of Respiratory Medicine, Tokyo Women's Medical University School of Medicine, Tokyo, Japan

\author{
Corresponding author: \\ Etsuko Tagaya \\ Department of Respiratory Medicine, Tokyo Women's Medical \\ University School of Medicine, \\ 8-1 Kawada-cho, Shinjuku-ku, Tokyo 162-8666, Japan \\ E-mail: tagaya.etsuko@twmu.ac.jp
}

\section{Introduction}

Asthma is a heterogeneous chronic inflammatory disease affecting airways in the lung. The main symptoms of asthma are defined as wheezes, shortness of breath, chest tightness and cough that vary over time. ${ }^{1}$ Asthma comprises a number of different clinical phenotypes and molecular endotypes. Most asthma patients, approximately 350 million individuals worldwide, can manage their symptoms by using conventional therapies, such as inhaled corticosteroids (ICS) and long-acting beta 2 -agonists (LABA). ${ }^{2}$ However, approximately $4 \%$ of asthma patients do not achieve disease control, which is defined as severe asthma, and these patients may receive additional therapies based on disease phenotypes, including oral corticosteroids (OCS), bronchial thermoplasty, or molecular-targeted biologic drugs. ${ }^{3-5}$ For severe asthma patients with type-2 inflammation, there are currently four biologic drugs (omalizumab, mepolizumab, benralizumab, and dupilumab) available in Japan. Each of these biologic drug have been shown to improve asthma control in terms of the reduction of asthma exacerbation rates and/or OCS use. ${ }^{6-10} \mathrm{Im}$ portantly, patients with certain asthma phenotypes, such as allergic or eosinophilic, may be more responsive to treatment with biologics. Additionally, these biologic drugs are effective 
not only for asthma, but also for other allergic and eosinophil-associated diseases such as allergic rhinitis, allergic dermatitis, and chronic rhinosinusitis, which are often comorbid with asthma. ${ }^{11-13}$

To date, very few studies have directly compared the effect of biologic drugs, and there is no definite indication for the selection of biologic drugs. Although most patients showed a favorable response to biologic drugs, some may need to discontinue one treatment and change to another biologic drug. There are no reports on the outcomes and clinical characteristics of patients after treatment failure with an initial biologic drug. Therefore, we sought to evaluate the characteristics and outcomes of patients treated with multiple biologic drugs.

\section{Methods}

This study was conducted at Tokyo Women's Medical University Hospital where biologic drugs are widely used. We retrospectively reviewed the medical records of patients with severe asthma (age 20 years or over) who received biologic drugs between May 2009 and September 2019. During this study period, four biologic drugs were commercially available: omalizumab from May 2009, mepolizumab from June 2016, benralizumab from April 2018, and dupilumab from March 2019. If patients met the indication criteria, physicians could prescribe a biologic drug to their patients; selection of a particular biologic drug was made based on the physician's discretion and commercial availability. Severe asthma was defined as requiring regular treatment with beclomethasone dipropionate (> $1000 \mu \mathrm{g} /$ day) or an equivalent dose of ICS, and other additional asthma medications, such as a leukotriene receptor antagonist, long-acting muscarinic antagonist, theophylline, antihistamine, and OCS (equivalent to guideline steps 4 or 5, according to the Global Initiative for Asthma 2019). ${ }^{3}$ Patients received biologic drugs for at least 16 weeks, except in cases where discontinuation was necessary due to side effects. We considered switching biologic drugs when patients' asthma symptoms persisted or if they could not discontinue or de-escalate daily dose of OCS. After discontinuation of the biologic drug, the duration before switching to another biologic drug was set at least 16 weeks. Patients who received omalizumab were allergic to at least one perennial allergen, as confirmed via a blood test or prick test. Both mepolizumab and benralizumab were used for patients with a peripheral blood eosinophil count of $>150 / \mu \mathrm{L}$. Patients who received dupilumab were recruited irrespective of a minimum peripheral blood eosinophil counts or biomarkers of type-2 inflammation. Patients with allergic bronchopulmonary aspergillosis and eosinophilic granulomatosis with polyangiitis, were excluded from this study.

The data collected from each patient included age, sex, body mass index (BMI), peripheral blood eosinophil counts, total IgE levels, fractional exhaled nitric oxide (FeNO), disease comorbidities, smoking history, regular OCS use, and the type of biologic drugs used during the study period. In addition, asthma phenotype such as type 2 asthma, atopic asthma, eosinophilic asthma, and overlap were evaluated based on peripheral blood eosinophil counts $(>300 / \mu \mathrm{L})$ and allergic status (1 or more perennial inhaled allergen-specific IgE concentrations of more than $0.70 \mathrm{UA} / \mathrm{mL}) .{ }^{14}$ In this study, type 2 asthma was defined as asthma with eosinophilic and/ or atopic status. The reason for the change in biologic drugs and the type of the latest biologic drug used, were reviewed. We also evaluated changes in annualized asthma exacerbation rates before and after the use of the final biologic drug introduced. An asthma exacerbation event was defined as the deterioration of asthma maintenance that required hospital admission or an emergency department visit, and treatment with systemic corticosteroids. The annualized exacerbation rate was defined as the number of exacerbations $\times 12 /$ the $\mathrm{du}-$ ration of the observation period (months). In addition, we confirmed changes in the asthma control test (ACT) scores and OCS dose from before and one year, after the initiation of treatment with the final biologic drug. ${ }^{15}$ ACT scores of 2025 were classified as well-controlled asthma. In addition, the minimal clinically important difference (MCID), defined as a three-point increase in ACT score, was considered reflective of a clinical improvement. ${ }^{16}$ The OCS dose was described as the equivalent prednisolone dose.

The institutional review board of Tokyo Women's Medical University approved the study (approval number: 5593), and all procedures were performed in accordance with the Good Clinical Practice guidelines and the Declaration of Helsinki. The study protocol was registered in the WHO International Clinical Trials Registry Platform (ICTRP) with the registration number UMIN000043274. Written informed consent was obtained from the patients at the initiation of the biologic drug treatment.

Data for continuous variables were expressed as the median (interquartile range) and for categorical variables as a percentage. Continuous variables were compared using the Mann-Whitney $U$ test and categorical variables with the Fisher's exact test. The paired $t$-test was used to compare the variables before and after the biologic drug treatment. A $p$-value $<0.05$ was considered statistically significant. Analyses were performed using JMP software v11.0 (SAS Institute Japan Ltd., Tokyo, Japan).

\section{Results \\ Characteristics of patients}

A total of 105 patients were enrolled in this study. Eightyfive patients received one biologic drug, while 20 patients received more than two biologic drugs (Figure 1). Among these 20 cases, 12 patients received two biologic drugs, 6 received three biologic drugs, and 2 received four biologic drugs (Table 1). Compared to the single biologic drug use group, patients who received multiple biologic drugs showed significantly higher FeNO levels ( $p=0.02$ ) (Table 2). In addition, the multiple biologic drugs group had a significantly higher proportion of comorbidities, including allergic rhinitis and chronic rhinosinusitis with nasal polyps (CRSwNP) $(p=0.02$ and $p<0.001$, respectively). No significant differences in any other parameters, such as age, sex, peripheral blood eosinophil counts, and total IgE levels, were observed. All patients who received multiple biologic drugs were type 2 phenotype and $80 \%$ of them overlapped atopic and eosinophilic status. 


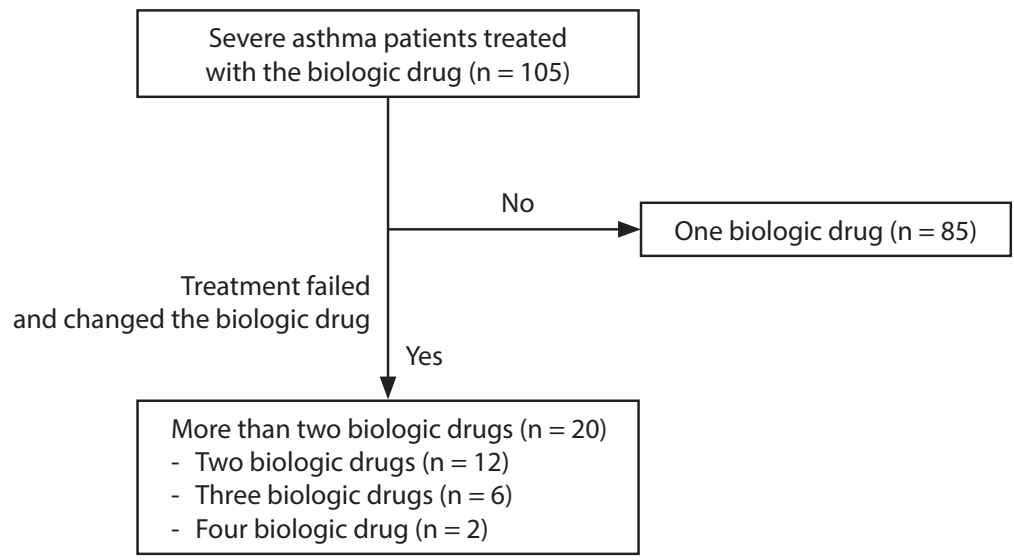

Figure 1. The number of severe asthma patients who the received biologic drug.

Table 1. Clinical characteristics of patients who received multiple biologic drugs.

\begin{tabular}{|c|c|c|c|c|c|c|c|c|c|}
\hline & Biologics 1 & Biologics 2 & Biologics 3 & Biologics 4 & Phenotype & $\begin{array}{c}\text { Blood } \\
\text { eosinophil }\end{array}$ & $\begin{array}{l}\text { Total } \\
\text { IgE }\end{array}$ & FeNO & Comorbidities \\
\hline $82 \mathrm{~F}$ & Omalizumab & Mepolizumab & - & - & Overlap & 523 & 353 & 137 & allergic rhinitis, CRSsNP \\
\hline $43 \mathrm{~F}$ & Omalizumab & Dupilumab & - & - & Atopic & 38 & 106 & 18 & $\begin{array}{l}\text { allergic rhinitis, atopic } \\
\text { dermatitis }\end{array}$ \\
\hline $66 \mathrm{M}$ & Omalizumab & Benralizumab & - & - & Overlap & 304 & 362 & 70 & allergic rhinitis, CRSwNP \\
\hline $79 \mathrm{M}$ & Omalizumab & Mepolizumab & - & - & Overlap & 341 & 37 & 123 & COPD (ACO) \\
\hline $62 \mathrm{M}$ & Omalizumab & Mepolizumab & - & - & Overlap & 696 & 556 & 94 & CRSwNP, EOM \\
\hline $41 \mathrm{~F}$ & Omalizumab & Mepolizumab & - & - & Overlap & 701 & 191 & 84 & EOM \\
\hline $81 \mathrm{~F}$ & Omalizumab & Mepolizumab & - & - & Overlap & 302 & 354 & 151 & allergic rhinitis, CRSsNP \\
\hline $47 \mathrm{~F}$ & Omalizumab & Mepolizumab & - & - & Atopic & 158 & 87 & 62 & allergic rhinitis \\
\hline $67 \mathrm{M}$ & Omalizumab & Mepolizumab & - & - & Overlap & 713 & 42 & 111 & CRSwNP, EOM \\
\hline $60 \mathrm{~F}$ & Omalizumab & Dupilumab & - & - & Overlap & 538 & 594 & 182 & $\begin{array}{l}\text { CRSwNP, atopic dermatitis, } \\
\text { spontaneout urticaria }\end{array}$ \\
\hline $54 \mathrm{M}$ & Omalizumab & Mepolizumab & - & - & Overlap & 377 & 272 & 16 & allergic rhinitis \\
\hline $49 \mathrm{M}$ & Omalizumab & Dupilumab & - & - & Overlap & 1310 & 517 & 161 & $\begin{array}{l}\text { allergic rhinitis, spontaneous } \\
\text { urticaria }\end{array}$ \\
\hline $57 \mathrm{~F}$ & Omalizumab & Mepolizumab & Benralizumab* & - & Overlap & 581 & 517 & 92 & CRSwNP \\
\hline $40 \mathrm{~F}$ & Benralizumab & Mepolizumab & Omalizumab & - & Atopic & 261 & 218 & 84 & allergic rhinitis, CRSwNP \\
\hline $41 \mathrm{M}$ & Omalizumab & Mepolizumab & Benralizumab & - & Overlap & 1080 & 154 & 31 & $\begin{array}{l}\text { allergic rhinitis, CRSwNP, } \\
\text { EOM }\end{array}$ \\
\hline $27 \mathrm{~F}$ & Benralizumab & Omalizumab & Dupilumab & - & Overlap & 321 & 884 & 46 & allergic rhinitis \\
\hline $50 \mathrm{~F}$ & Omalizumab & Benralizumab & Mepolizumab & - & Atopic & 169 & 208 & 20 & allergic rhinitis, CRSsNP \\
\hline $55 \mathrm{M}$ & Mepolizumab & Benralizumab & Omalizumab & - & Overlap & 309 & 201 & 257 & $\begin{array}{l}\text { allergic rhinitis, CRSsNP, } \\
\text { spontaneous urticaria }\end{array}$ \\
\hline $47 \mathrm{~F}$ & Omalizumab & Mepolizumab & Omalizumab $^{*}$ & Dupilumab & Overlap & 397 & 18 & 24 & $\begin{array}{l}\text { allergic rhinitis, atopic } \\
\text { dermatitis }\end{array}$ \\
\hline $62 \mathrm{M}$ & Omalizumab & Dupilumab & Benralizumab & Mepolizumab $^{*}$ & Overlap & 489 & 210 & 31 & $\begin{array}{l}\text { CRSwNP, EOM, COPD } \\
\text { (ACO) }\end{array}$ \\
\hline
\end{tabular}

Abbreviations: EOM, eosinophilic otitis media; CRSwNP, chronic sinusitis with nasal polyps; CRSsNP, chronic sinusitis without nasal polyps; ACO, Asthma-COPD overlap;

*rechallenge, "no preferable effect was observed after the latest biologic drug use 
Table 2. Baseline characteristics at the initiation of the biologic drug.

\begin{tabular}{|c|c|c|c|}
\hline Patient's characteristics & $\begin{array}{l}\text { patients who received } \\
\text { single biologic drug } \\
\qquad(\mathrm{n}=\mathbf{8 5})\end{array}$ & $\begin{array}{l}\text { patients who received } \\
\text { multiple biologic drugs } \\
\qquad(\mathrm{n}=20)\end{array}$ & $p$ \\
\hline Age (years) & $58(45-66)$ & $54.5(46-63)$ & $0.95^{\ddagger}$ \\
\hline Male (\%) & $26(31)$ & $9(45)$ & $0.22^{\dagger}$ \\
\hline FENO (ppb) & $30(18-72)$ & $84(31-127)$ & $0.02^{\ddagger}$ \\
\hline OCS use & $34(40)$ & $6(30)$ & $0.41^{\dagger}$ \\
\hline Blood eosinophil counts $(/ \mu \mathrm{L})$ & $227(98-508)$ & $387(233-610)$ & $0.12^{\ddagger}$ \\
\hline Total IgE (IU/mL) & $341(105-425)$ & $214(141-401)$ & $0.71^{\ddagger}$ \\
\hline BMI $\left(\mathrm{kg} / \mathrm{m}^{2}\right)$ & $23.9(20.9-26.7)$ & $25.1(21.2-27.4)$ & $0.42^{\ddagger}$ \\
\hline Smoking & $27(31)$ & $8(42)$ & $0.48^{\dagger}$ \\
\hline \multicolumn{4}{|l|}{ Comorbidities } \\
\hline - allergic rhinitis & $31(36.5)$ & $13(65)$ & $0.02^{\dagger}$ \\
\hline - EOM & $8(10)$ & $5(25)$ & $0.12^{\dagger}$ \\
\hline - CRSwNP & $6(7)$ & $8(40)$ & $<0.001^{\dagger}$ \\
\hline - CRSsNP & $6(7)$ & $4(20)$ & $0.09^{\dagger}$ \\
\hline - spontaneous urticaria & $5(6)$ & $2(10)$ & $0.62^{\dagger}$ \\
\hline - atopic dermatitis & $6(7)$ & $3(15)$ & $0.37^{\dagger}$ \\
\hline - COPD & $10(12)$ & $2(10)$ & $1^{\dagger}$ \\
\hline \multicolumn{4}{|l|}{ Biologic drugs at the initiation } \\
\hline - omalizumab & $67(84)$ & $17(85)$ & $0.76^{\dagger}$ \\
\hline - mepolizumab & $9(11)$ & $2(10)$ & $0.16^{\dagger}$ \\
\hline - benralizumab & $3(4)$ & $0(0)$ & $1^{\dagger}$ \\
\hline - dupilumab & $6(7)$ & $1(5)$ & $1^{\dagger}$ \\
\hline
\end{tabular}

Abbreviations: EOM, eosinophilic otitis media; CRSwNP, chronic sinusitis with nasal polyps; CRSsNP, chronic sinusitis without nasal polyps Values are median (interquartile range) or number (\%)

${ }^{\dagger}$ Fisher's exact test, "Mann-Whitney Utest

Table 3. The reason for the change of biologic drugs.

\begin{tabular}{|c|c|c|c|c|}
\hline & No improvement ${ }^{*}$ & Comorbidities" & Side effect ${ }^{*}$ & $\begin{array}{l}\text { The number } \\
\text { of biologics } \\
\text { changed }^{*}\end{array}$ \\
\hline omalizumab & 14 & 11 & 0 & 19 \\
\hline mepolizumab & 4 & 2 & 1 & 5 \\
\hline benralizumab & 4 & 1 & 1 & 4 \\
\hline dupilumab & 1 & 0 & 0 & 1 \\
\hline
\end{tabular}

${ }^{*}$ Cumulative total number of patients

"Multiple choice allowed and there is some overlapping. 


\section{Reasons for biologic drug change}

Patients who did not respond to omalizumab treatment ( $n=19$ ) discontinued biologic drugs due to no preferable effect $(73.7 \%)$ or uncontrolled comorbidities (57.9\%) (Table 3). Most patients who had been treated with and discontinued mepolizumab $(n=5)$, benralizumab $(n=4)$, or dupilumab $(\mathrm{n}=1)$, changed biologic drugs due to uncontrolled asthma symptoms. In this study, approximately half (48.3\%) of drug discontinuation occurred due to the poor control of comorbidities. Only two patients changed biologic drugs because of side effects (one patient discontinued treatment due to skin rash, the other due to headache and fatigue).

\section{The latest biologic drug type and treatment outcomes}

Among patients who received multiple biologic drugs, 17 patients (85\%) received omalizumab, 2 patients (10\%) received mepolizumab, and 1 patient (5\%) received dupilumab at the initiation of treatment (Table 1). After treatment failure

a.

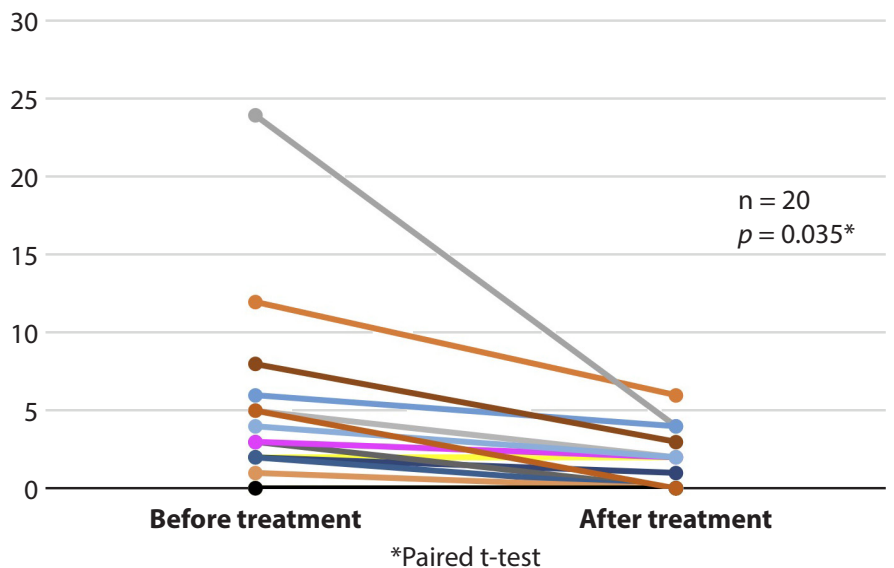

with the initial biologic drug, 2 patients were finally switched to omalizumab, 10 patients were switched to mepolizumab, 3 patients were switched to benralizumab, and 5 were switched to dupilumab.

Regarding treatment outcomes, 12 patients $(60 \%)$ showed a reduction in the number of annualized exacerbations $(p=$ 0.035) (Figure 2a). With regard to changes in the ACT score, 10 patients $(50 \%)$ achieved disease control 1 year after the initiation of the latest biologic drug (Figure 2b). Moreover, 12 patients (60\%) achieved MCID. The total difference between before and after treatment was also significant $(p<0.001)$. Lastly, we confirmed that five patients (25\%) showed a reduction in the OCS dose (Figure 2c). Although the OCS dose increased in 1 patient and 14 patients did not use OCS regularly, the reduction in the OCS dose before and after treatment was statistically significant $(p=0.038)$. Among patients who received multiple biologic drugs, most achieved disease control of allergic comorbidities as well as their asthma.

b.

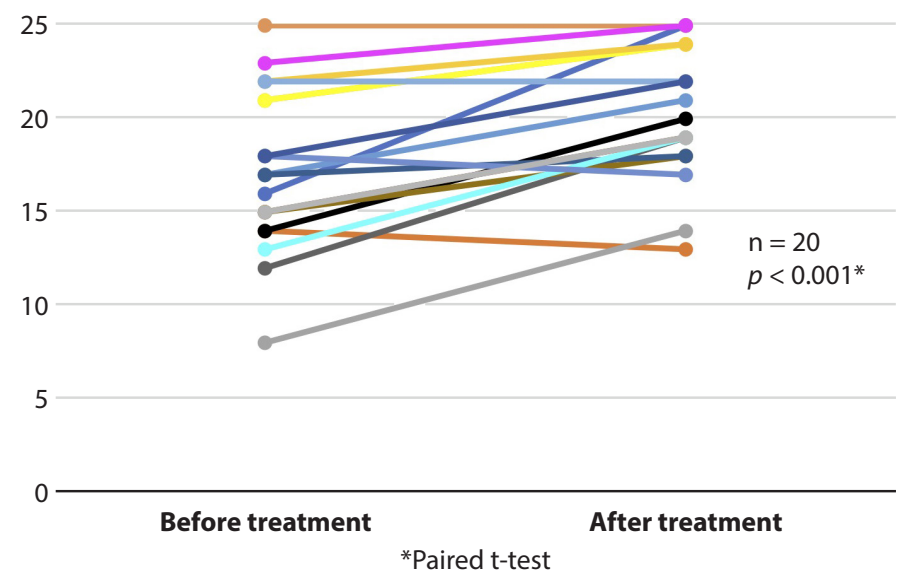

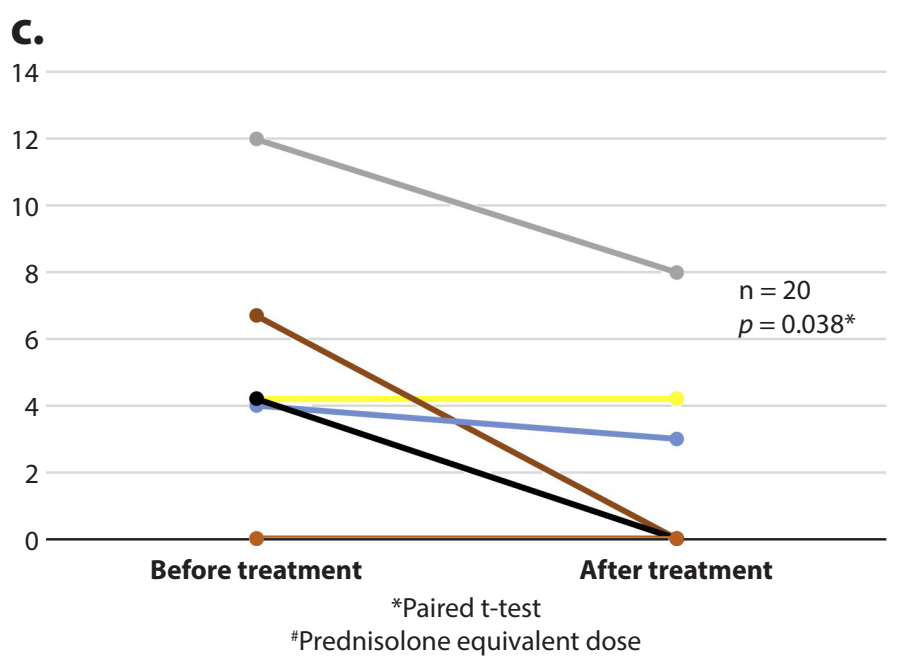

Figure 2 .

Figure 2a. Annualized exacerbation rates before and after treatment of the latest biologic drug.

Figure $2 \mathrm{~b}$. ACT scores before and after treatment of the latest biologic drug.

Figure 2c. OCS dose before and after treatment of the latest biologic drug. 


\section{Discussion}

In this study, we revealed the clinical characteristics and treatment outcomes of patients with severe asthma, who received treatment with more than two biologic drugs. Because only 20 patients who received multiple biologic drugs were evaluated, it seems rather difficult to reach a definite conclusion. Therefore, we regarded this study as a pilot study and future research with a higher number of participants is much needed. This study showed that patients who tended to have a significantly higher proportion of allergic or eosinophilic airway comorbidities, experienced increased treatment failure with biologic drugs. Additionally, we confirmed that patients with the most severe asthma who were treated with multiple biologic drugs, achieved disease control even if treatment with an initial biologic drug had failed.

Most clinical characteristics of patients with severe asthma who used multiple biologic drugs did not differ from those of patients who used only one biologic drug. Only FeNO and the proportion of allergic and eosinophilic airway comorbidities tended to be higher in the multiple biologic drugs group. One possible explanation for this is that a higher FeNO level may reflect the existence of allergic or eosinophilic airway comorbidities. ${ }^{17,18}$ Both allergic rhinitis and CRSwNP, often comorbid with asthma, are associated with type-2 airway inflammation. ${ }^{19,20}$ In this study, a considerable number of patients changed biologic drugs due to uncontrolled comorbidities. The "one airway, one disease" theory shows that the treatment of both asthma and airway comorbidities is essential. ${ }^{21}$ Therefore, the selection of a biologic drug treatment should be made likewise. For example, it has been reported that patients with severe asthma and CRSwNP, showed a positive treatment response to dupilumab..$^{22}$ Similarly, symptoms of patients with asthma and eosinophilic otitis media, were well controlled by mepolizumab. ${ }^{23}$ Moreover, various reports show that other allergic diseases can be treated by biologic drugs. For example, omalizumab is effective for spontaneous urticaria, and dupilumab has shown favorable effects in the treatment of atopic dermatitis. ${ }^{12,24}$ The results from this study showed that disease comorbidities may contribute to the treatment failure with biologic drugs. Therefore, allergic and eosinophilic airway disease comorbidities should be taken into consideration when selecting a particular biologic drug treatment. Optimal disease control can be achieved by treating both asthma and its associated comorbidities.
This study revealed that a significantly higher number of patients who used multiple biologic drugs ultimately achieved disease control. To the best of our knowledge, there has been no direct comparison between biologic drugs. An arm-based network meta-analysis did not show significant superiority of one biologic drug over the other. ${ }^{25}$ However, indirect comparison between dupilumab and benralizumab showed dupilumab significantly reduced annual exacerbation rates than benralizumab. ${ }^{26}$ In addition, several studies showed that patients who did not achieve symptom maintenance with the initial biologic treatment, achieved disease control when they switched to other biologics. ${ }^{27,28}$ In Japanese populations, it has been reported that $80 \%$ of difficult-to-treat asthma is of the Th2 phenotype. ${ }^{14}$ Among these cases, approximately 35\% overlap between allergic and eosinophilic phenotypes. Interestingly, in this study, $80 \%$ of patients with multiple biologic drug use showed overlap between atopic status and high eosinophilic condition. Therefore, it is possible that more than two biologics can be potential candidates for the treatment of these patients (Figure 3). Because we still do not have a definitive procedure for the selection of biologic drugs, we should choose and switch candidate biologics based on allergic comorbidities and asthma subtype. We confirmed that all treatment assessment data, namely annualized asthma exacerbation rates, ACT scores, and OCS use, significantly improved after the initiation of the final biologic drug. These findings suggest that a patient's biologic drug should potentially be switched if a patient does not achieve favorable results and that changing the biologic drug may lead to better treatment outcomes.

This study has several limitations that should be acknowledged. First, the selection of biologic drugs can be influenced by the availability of drugs. In Japan, the anti-IgE antibody, omalizumab, was only available from 2009 to 2015, after which the anti-interleukin (IL) 5 antibody mepolizumab, anti-IL-5R $\alpha$ antibody benralizumab, and anti-IL4/13 antibody dupilumab became commercially available, in that order. Moreover, the selection of different biologic drugs ultimately depends on each physician's discretion. This may introduce a selection bias when choosing a biologic drug. However, because biologic drugs are used on the basis of commercial indications, the evaluation of disease comorbidities and treatment outcomes after multiple biologic drug use is warranted.

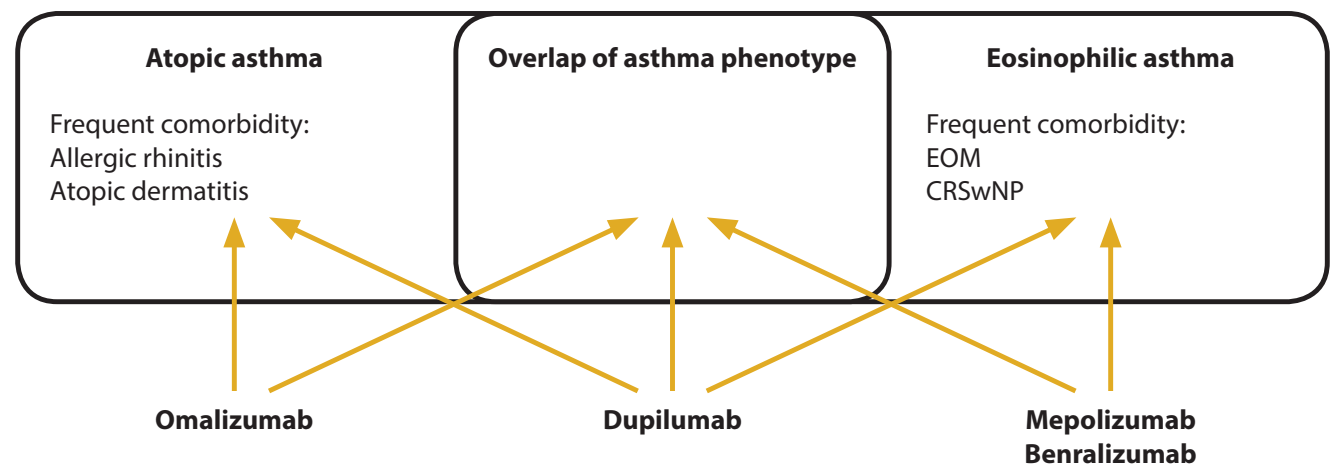

Figure 3. Asthma phenotype of patients and possible treatment suggestion. 
Finally, since this study was a single-center retrospective analysis, the results might not be applicable to all patients. In particular, the severity of asthma phenotype may vary by region and race. ${ }^{29}$ To validate these biases, a further study evaluating the four available biologic drugs at the initiation of the study, with a larger number of study participants and a strict protocol, is needed.

\section{Conclusions}

The results from this pilot study suggest that assessment of allergic and eosinophilic airway comorbidities is important in the selection of biologics. When the patient does not achieve disease control with treatment of the initial biologic drug, changing treatment with other biologic drugs may be a good option. The optimal choice of a biologic drug may contribute to good treatment outcomes with regard to both asthma and associated comorbidities.

\section{References}

1. Braido F, Tiotiu A, Kowal K, Mihaicuta S, Novakova P, Oguzulgen IK. Phenotypes/endotypes-driven treatment in asthma. Curr Opin Allergy Clin Immunol. 2018;18(3):184-9.

2. GBD 2015 Chronic Respiratory Disease Collaborators. Global, regional, and national deaths, prevalence, disability-adjusted life years, and years lived with disability for chronic obstructive pulmonary disease and asthma, 1990-2015: a systematic analysis for the Global Burden of Disease Study 2015. Lancet Respir Med. 2017;5(9):691-706.

3. Global Initiative for Asthma [Internet]. Fontana: Global Initiative for Asthma; c2020 [cited 2020 Oct 1]. Diagnosis and Management of Difficult-to-treat and Severe Asthma; [about 1 screen]. Available from: https://ginasthma.org/severeasthma/.

4. Hekking PP, Wener RR, Amelink M, Zwinderman AH, Bouvy ML, Bel $\mathrm{EH}$. The prevalence of severe refractory asthma. J Allergy Clin Immunol. 2015;135(4):896-902.

5. Castro M, Rubin AS, Laviolette M, Fiterman J, De Andrade Lima M, Shah PL, et al. Effectiveness and safety of bronchial thermoplasty in the treatment of severe asthma: a multicenter, randomized, double-blind, sham-controlled clinical trial. Am J Respir Crit Care Med. 2010;181(2): 116-24.

6. Normansell R, Walker S, Milan SJ, Walters EH, Nair P. Omalizumab for asthma in adults and children. Cochrane Database Syst Rev. 2014(1): Cd003559.

7. Busse W, Corren J, Lanier BQ, McAlary M, Fowler-Taylor A, Cioppa $\mathrm{GD}$, et al. Omalizumab, anti-IgE recombinant humanized monoclonal antibody, for the treatment of severe allergic asthma. J Allergy Clin Immunol. 2001;108(2):184-90.

8. Bel EH, Wenzel SE, Thompson PJ, Prazma CM, Keene ON, Yancey SW, et al. Oral glucocorticoid-sparing effect of mepolizumab in eosinophilic asthma. N Engl J Med. 2014;371(13):1189-97.

9. Bleecker ER, FitzGerald JM, Chanez P, Papi A, Weinstein SF, Barker P, et al. Efficacy and safety of benralizumab for patients with severe asthma uncontrolled with high-dosage inhaled corticosteroids and long-acting $\beta$ (2)-agonists (SIROCCO): a randomised, multicentre, placebo-controlled phase 3 trial. Lancet. 2016;388(10056):2115-27.

10. Rabe KF, Nair P, Brusselle G, Maspero JF, Castro M, Sher L, et al. Efficacy and Safety of Dupilumab in Glucocorticoid-Dependent Severe Asthma. N Engl J Med. 2018;378(26):2475-85.
11. Casale TB, Condemi J, LaForce C, Nayak A, Rowe M, Watrous M, et al. Effect of omalizumab on symptoms of seasonal allergic rhinitis: a randomized controlled trial. JAMA. 2001;286(23):2956-67.

12. Beck LA, Thaçi D, Hamilton JD, Graham NM, Bieber T, Rocklin R, et al. Dupilumab treatment in adults with moderate-to-severe atopic dermatitis. N Engl J Med. 2014;371(2):130-9.

13. Maspero JF, Katelaris CH, Busse WW, Castro M, Corren J, Chipps BE, et al. Dupilumab Efficacy in Uncontrolled, Moderate-to-Severe Asthma with Self-Reported Chronic Rhinosinusitis. J Allergy Clin Immunol Pract. 2020;8(2):527-39.e9.

14. Matsusaka M, Fukunaga K, Kabata H, Izuhara K, Asano K, Betsuyaku T. Subphenotypes of type 2 severe asthma in adults. J Allergy Clin Immunol Pract. 2018;6(1):274-6.e2.

15. Nathan RA, Sorkness CA, Kosinski M, Schatz M, Li JT, Marcus P, et al. Development of the asthma control test: a survey for assessing asthma control. J Allergy Clin Immunol. 2004;113(1):59-65.

16. Schatz M, Kosinski M, Yarlas AS, Hanlon J, Watson ME, Jhingran P. The minimally important difference of the Asthma Control Test. J Allergy Clin Immunol. 2009;124(4):719-23.e1.

17. Ciprandi G, Gallo F, Ricciardolo FL, Cirillo I. Fractional Exhaled Nitric Oxide: A Potential Biomarker in Allergic Rhinitis? Int Arch Allergy Immunol. 2017;172(2):99-105.

18. Asano T, Takemura M, Kanemitsu Y, Yokota M, Fukumitsu K, Takeda N, et al. Combined measurements of fractional exhaled nitric oxide and nasal nitric oxide levels for assessing upper airway diseases in asthmatic patients. J Asthma. 2018;55(3):300-9.

19. Bousquet J, Schünemann HJ, Samolinski B, Demoly P, Baena-Cagnani CE, Bachert C, et al. Allergic Rhinitis and its Impact on Asthma (ARIA): achievements in 10 years and future needs. J Allergy Clin Immunol. 2012;130(5):1049-62.

20. Canonica GW, Malvezzi L, Blasi F, Paggiaro P, Mantero M, Senna G, et al. Chronic rhinosinusitis with nasal polyps impact in severe asthma patients: Evidences from the Severe Asthma Network Italy (SANI) registry. Respir Med. 2020;166:105947.

21. Grossman J. One airway, one disease. Chest. 1997;111(2 Suppl):11-6s.

22. Bachert C, Han JK, Desrosiers M, Hellings PW, Amin N, Lee SE, et al. Efficacy and safety of dupilumab in patients with severe chronic rhinosinusitis with nasal polyps (LIBERTY NP SINUS-24 and LIBERTY NP SINUS-52): results from two multicentre, randomised, double-blind, placebo-controlled, parallel-group phase 3 trials. Lancet. 2019;394(10209): 1638-50.

23. Iino Y, Takahashi E, Ida S, Kikuchi S. Clinical efficacy of anti-IL-5 monoclonal antibody mepolizumab in the treatment of eosinophilic otitis media. Auris Nasus Larynx. 2019;46(2):196-203.

24. Maurer M, Rosén K, Hsieh HJ, Saini S, Grattan C, Gimenéz-Arnau A, et al. Omalizumab for the treatment of chronic idiopathic or spontaneous urticaria. N Engl J Med. 2013;368(10):924-35.

25. Edris A, De Feyter S, Maes T, Joos G, Lahousse L. Monoclonal antibodies in type 2 asthma: a systematic review and network meta-analysis. Respir Res. 2019;20(1):179.

26. Ando K, Tanaka A, Sagara H. Comparative Efficacy and Safety of Dupilumab and Benralizumab in Patients with Inadequately Controlled Asthma: A Systematic Review. Int J Mol Sci. 2020;21(3):889.

27. Bagnasco D, Menzella F, Caminati M, Caruso C, Guida G, Bonavia M, et al. Efficacy of mepolizumab in patients with previous omalizumab treatment failure: Real-life observation. Allergy. 2019;74(12):2539-41.

28. Pérez de Llano LA, Dacal Rivas D, Cosío BG. Mepolizumab and reslizumab, two different options for severe asthma patients with prior failure to omalizumab. Allergy. 2020;75(4):940-2.

29. Moore WC, Meyers DA, Wenzel SE, Teague WG, Li H, Li X, et al. Identification of asthma phenotypes using cluster analysis in the Severe Asthma Research Program. Am J Respir Crit Care Med. 2010;181(4): 315-23. 